[This document contains the author's accepted manuscript. For the publisher's version, see the link in the header of this document.]

\title{
Agreements and Disagreements in a Computer-Mediated Discussion
}

\author{
Nancy K. Baym \\ Submitted to Research on Language and Social Interaction May, 1996. \\ Nancy K. Baym is an Assistant Professor in the Department of Communication, \\ Wayne State University, Detroit, MI. \\ Author address: 585 Manoogian, \\ Wayne State University, \\ Detroit, MI 48202 \\ email: $\underline{\text { nbaym@cms.cc.wayne.edu }}$
}

Paper citation: Baym, N. K. (1996). Agreements and Disagreements in a Computer-Mediated Group. Research on Language and Social Interaction, Vol. 29, 315-346.

\begin{abstract}
:
Agreements and disagreements in a predominantly female computer-mediated discussion group which discusses soap operas are analyzed in terms of their message features. The findings differ from those on agreements and disagreements in oral interaction and in written letters. These agreements included qualifications, provision of reasoning, elaborations, and other features uncommon in oral and epistolary agreements. The disagreements were more mitigated than the agreements, but were more likely to contain direct contradictory assertions than agreements were to contain affirming assertions. They too were likely to include elaborations. These findings are attributed interactions between the medium, the topic under discussion, the context of media use, participant gender and the context participants strive to create.
\end{abstract}


Agreements and Disagreements in a Computer-Mediated Discussion

\author{
Introduction
}

Computer-mediated communication (CMC) is written, yet is marked by many features associated with face-to-face interaction. CMC's status as "a hybrid language variety displaying characteristics of both oral and written language" (Ferrara, Brunner, \& Whittemore, 1991, p. 10) raises the question of how language activities are shaped in this medium. This paper examines agreements and disagreements in one computermediated discussion group, contrasting them with what is known about those activities in oral conversation and letters. This discussion group, and the tens of thousands like it, are also a hybrid between mass and interpersonal communication, further complicating the medium's communicative activities. After a discussion of the medium, I examine agreements and disagreements in a Usenet newsgroup devoted to discussing soap operas. The analysis informs our understanding of language use in CMC and shows how media, participant characteristics, context, topic, and emergent contextual goals influence language use.

Usenet is an enormous collection of topically-organized discussion groups distributed through the Internet. It links millions of users at more than 100,000 sites across the United States and throughout most of the world (Reid, 1993). Most sites are computers at universities or colleges, computing and software companies, and scientific laboratories, both government and private. Increasingly people are gaining Usenet access through commercial networks such as America Online and Prodigy ${ }^{\circledR}$ and through community-organized Freenets.

Newsgroups are comprised of electronic letters called posts or articles which are contributed by individuals from private accounts at their sites. With the exceptions of 


\section{CMC responses -- 2}

some site restrictions and some moderated groups, these articles can be any length and are not censored prior to distribution. People read and post through programs called newsreaders, which keep track of which articles have already been read, allow people to edit what they will read, and allow people to reply to posts and create new lines of discussion. Most newsreaders also enable people to quote the posts to which they respond directly within their responses, usually marked with " $>$ " in the left margin.

When people read newsgroups, they see only the articles which have arrived at their sites since the last readings. Participants need not be on-line simultaneously; they can read and respond at different times. Messages are stored at each site for a time period left to the sites' system administrators to decide, usually no longer than two weeks. Until the old messages are removed, readers can check in at their convenience to read or respond to what has arrived.

\section{Usenet Discourse as a Hybrid Register}

The most natural comparison to Usenet would seem to be writing, rather than speaking. In written media, including Usenet, most of the nonverbal cues of oral interactions are not available. There can be no body movements, vocal tone, rate or volume. However, some paralinguistic cues are available, as writers on Usenet, like those in other forms of writing, exploit "aspects of graphic form such as spelling, punctuation, typography, and layout, for expressive purposes" (Danet, 1993).

The single greatest difference between writing and oral interaction, especially face-to-face conversation, is that participants do not share a common physical or temporal context. As a result, writing is generally more explicit than oral language, as it must make manifest all that would be apparent from context. Ong (1982), for instance, argues that: 


\section{CMC responses -- 3}

To make yourself clear without gesture, without facial expression, without intonation, without a real hearer, you have to foresee circumspectly all possible meanings a statement may have for any possible reader in any possible situation, and you have to make your language work so as to come clear all by itself, with no existential context. (p. 104)

Chafe (1982) too argues that writers seek to produce texts which will make sense to diverse audiences reading at different times and in different places. He argues this makes writing non-interactive, saying "I will speak of 'involvement' with the audience as typical for a speaker, and 'detachment' from the audience as typical for a writer" (p. 45).

Chafe (1982) also posits that decontextualization and the slower pace of writing lead to greater closure than is found in oral utterances:

in writing, it would seem, our thoughts must constantly get ahead of our expression of them in a way to which we are totally unaccustomed when we speak [...] The result is that we have time to integrate a succession of ideas into a single linguistic whole in a way that is not available in speaking. (pg. 37)

Also connecting writing with non-interactivity and closure, Ong (1982) argues that "the author cannot be reached in any book [...] After absolutely total and devastating refutation, it says exactly the same thing as before" (pg. 79).

Some of these elements of writing are true of Usenet discourse. Usenet messages lack much of the context available in oral interaction; writers cannot depend on their readers to have heard prior messages, to catch nonverbal nuance or to have the same 


\section{CMC responses -- 4}

objects in their range of sight. As a result there is greater pressure on the Usenet writer than on the conversationalist to make referents and implied meanings explicit.

However, like speech, Usenet messages are contextualized and interactive in many ways. Though it is asynchronous, there is more of a shared temporal context than there is in print media, since no sites carry messages indefinitely. There is thus no need for Usenet messages to be timeless. There is also a similar physical context. Although the locations of readers differs, writers know that their readers will be seated at computers. Often posts will refer to the computer, acknowledging and invoking this mutual context.

Usenet groups also share referents, discourse histories and normative conventions, which are possible because the groups are ongoing. As Wilkins (1991) found in studying $\mathrm{CMC}$, computer users created not just a context, but a community through their messages, a phenomenon she attributed to their use of personal names, the dyadic nature of the conversation, the regular presence of disfluencies in $\mathrm{CMC}$, and the creation of new spheres of shared knowledge and norms. Baym (1995) has also elaborated how users of Usenet newsgroups can strategically appropriate shared resources to verbally create and sustain rich communities.

Furthermore, Usenet writers are not completely distanced from the readers. It is true that Usenet writers do not know everyone who will read their messages. They can not see their readers respond, so they can't adjust their messages during their production to accommodate reader responses. However, they may know some of their likely readers from previous discourse. If they have been participating in a group for some time, they may be able to predict how particular others will react to their messages. Writers are also able to respond to their readers; while a sent post cannot be revised, Usenet writers can be and are reached. All messages include the senders' email 


\section{CMC responses -- 5}

addresses and the writers can be and often are contacted, either through private email or through the group. Usenet involves continued interactions between writers and readers in which positions are affirmed and challenged, clarified and modified.

In these ways, Usenet is more like conversation than like writing. One of the most significant differences between oral interaction and Usenet, however, is that writers and readers are temporally separated. Messages are distributed through complex networks of sites, and may show up at different sites at different times. Conversation analysts (e.g. Heritage \& Atkinson, 1984) have argued that conversational coordination is achieved through turn sequences in which each turn displays understanding of the prior turn and projects possible next turns. While participants in oral conversation orient to prior turns, Usenet participants are not engaged synchronously and may not read or have access to all the material posted. Usenet writers cannot assume that all readers are aware of prior turns or even that other participants in a turn sequence will see their response. This is a fundamental difference in orientation between Usenet and conversation.

Furthermore, the temporal separation changes the meanings of many phenomena noted in oral turn-taking sequences. For example, while Levinson (1983) and Pomerantz (1984) argue that hesitations mark forthcoming dispreferred responses, all messages in Usenet are preceded by hesitation. Responses are routinely posted hours or days after the message to which they respond, a fact which reflects the nature of the medium rather than the response. One cannot therefore assume that the meanings of specific message features will transfer directly from conversational interaction to Usenet.

A final critical difference between oral conversation and Usenet stems from its status as both interpersonal and mass. While group participants may come to know one 


\section{CMC responses -- 6}

another as individuals, their messages are essentially broadcast to large audiences, most of whom they do not know. Usenet writing is thus never dyadic, even when replies are ostensibly addressed to particular individuals. This generates another fundamental difference in orientation. While speakers in oral interaction are accountable primarily to their conversational partners, Usenet interactants are responsible to the whole group. A personalized reply intended for only one reader is inappropriate as it offers nothing to the other readers. Indeed, participants always have the option of private email. When posted discussions do become exclusively dyadic, participants are likely to request that the pair "take it to email." On occassions when email bounces, it is common to see subject lines flagging a specific recipient and apologies for using the group to reach an individual.

Usenet is interpersonal in that messages may have consequences for dyadic relationships. However, those same messages also impact the social definition of the group and the public images of the interactants. For instance, if one person writes a nasty response to another's message, the result is not just the creation of animosity between two people, but the creation of a hostile group environment and a potential loss of public face for either or both posters involved in the exchange. Because discourse is the only means of creating social context in CMC, the messages play a particularly direct role in defining the group's social world and its inhabitants. It takes very few participants to upset or redefine a group's social environment.

While Usenet is unusual in its mix of mass and interpersonal communication, it is not alone in bridging between characteristics of written and oral language norms. Both speech and writing can take many forms depending on the social contexts in which they are embedded. These differences between oral and written language are extremes on a continuum. As Biber (1986) writes, there are "few (if any) absolute differences [...] between speech and writing" (p. 385). The hybrid form most like Usenet 


\section{CMC responses -- 7}

is the letter, which is also near the center of the written-oral continuum. Basso (1974) shows that letters are situated in relational and cultural contexts which shape what is and is not appropriate. Mulkay (1986) shows that letters are like conversation in that they "employ direct, personal address; they require participants to respond appropriately to others' contributions; they involve a turn-taking sequence; and several contributors can take part" (p. 303). In contrast to spoken language, turns in letters are not temporally contingent, they are longer than spoken turns, make their topics more explicit, and cover more topics (Mulkay, 1986). Usenet posts too may carry direct address (though far less frequently than letters), may involve turn-taking sequences, involve multiple participants, are often longer than most spoken turns, make topics explicit and often cover multiple topics. In contrast to letters, however, Usenet messages' status as one of many posts addressed to multiple participants also means that there are no designated respondents and not all messages will receive responses. This can be compared to the oral phenomenon in which some topic initiators are not taken up by the other conversationalists.

To summarize, Usenet interaction is a hybrid between oral, written, interpersonal and mass communication. Like writing, participants are temporally separated. Usenet writers cannot assume that all readers will have read the messages to which they respond nor that they will be able to tell to which message they are responding. Usenet's quotation system, which allows writers to replicate whole or partial prior turns within their own messages, helps to mitigate the potential non-coherence of Usenet discourse by explicitly marking topic and creating an orientation to specific prior turns. Quotation is also a strategic resource for writers, as they can edit selectively to create contexts for their responses. In addition to explicitly identifying topic, Usenet writers must make much of the information explicit that would be carried by the voice, gestures or other nonverbal cues in face-to-face conversation. Like speech, Usenet is 
contextualized and interactive. Writers can assume whom many of their readers will be, that they will share many referents, will be reading within a few days and will be able to respond. Messages are not necessarily closed, nor is their meaning removed from the writers or from their audience. Like interpersonal communication, messages may be built off the comments of particular individuals, have consequences for one-on-one relationships, and can be highly personalized. However, like mass communication, Usenet interaction is always written for a large audience and is affected by and affects the writers' public images and the group as a whole.

\section{Agreement and Disagreement in Oral Conversation}

It is to be expected that these features of Usenet would impact the performance of agreements and disagreements. Not only are there likely to be message features not seen in oral conversation, but features which appear in both kinds of interaction could serve different functions. The work on these activities in conversation provides a useful starting point for understanding their performance in CMC.

Using the concept of preference, Pomerantz (1984) shows that in ordinary friendly talk participants are oriented toward interpersonal coordination, and thus toward consensus. As a result, with some exceptions, when one participant offers an assessment, agreements are preferred responses and disagreements dispreferred (Pomerantz, 1984; Brown \& Levinson, 1987). As Pomerantz describes it, this preference system is revealed in each activity's formal structures and timing. Agreements are usually stated explicitly and without hesitation. In contrast, disagreements are more likely to be delayed between, within and across turns through hesitations, questioning repeats, requests for clarification, and the like (Pomerantz, 1984). Dispreference is also marked through prefaces; in the case of dispreferred disagreements, partial agreements are particularly common prefaces (Levinson, 1983; Pomerantz, 1984; Mulkay, 1985, 


\section{CMC responses -- 9}

1986; Sheldon, 1992). In short, though they may be upgraded or downgraded, agreements tend to be structurally simple and temporally immediate in comparison to disagreements, which tend to be delayed and marked in ways which minimize stated disagreement and work to maintain consensus (Pomerantz, 1984). Given the nature of Usenet interaction, one can't expect these findings to generalize to this medium. For instance, as I have suggested above, the temporal separation intrinsic to Usenet interaction means that both agreements and disagreements follow extended time delays. These delays speak only to the nature of the medium, not the kind of response. Similarly, both agreements and disagreements in Usenet could require explicit marking and prefaces since topic cannot be inferred from an immediately prior turn. Thus several important conversational markers of oral agreement and disagreement are not at play in the same ways in Usenet.

Kotthoff (1993) argues that the performance of agreement and disagreement depends not only on the prior turn, but on other contexts, including genre, institutional situation, and culture. She examined 12 dyadic discussions between German and Anglo-American students and lecturers which were framed as "disputes" rather than as ordinary conversation. She found that when people agreed during the course of these disputes, their agreements were delayed and complex in all the ways Pomerantz's disagreements were. Kotthoff (1993) concludes that "preference structures are preshaped by institutional requirements, which are not necessarily shared by everybody, and in turn help to create the institutional setting" (p. 196). Here she raises an essential point. Contexts influence how these activities are performed, but not all participants share understandings of those contexts' requirements. As a result, how these activities are enacted must be continually negotiated in interaction. Those negotiations in turn influence the social worlds in which agreements and disagreements gain their meaning. 
CMC responses -- 10

Mulkay $(1985,1986)$ addresses the influence of communication medium on the agreements and disagreements in his examination of 80 written letters of scholarly scientific exchanges. In this medium, he found that the restrictions on turn-taking and topic organization made agreements more complex. They were "more likely to involve a formal restatement of what is agreed as well as explicit reference to agreement and they are more likely to be placed after some kind of preface" (1986, p. 308). He also found that the disagreements were notably more complex than the agreements. They were more likely to be marked with prefaces (especially partial agreements) and other indicants of dispreference. The main way in which his findings on disagreement differ from those of Pomerantz is that the written disagreements were more likely to be stated in strong and explicit terms, leading him to suggest that it may be easier to disagree when writing than when talking.

Usenet offers an exciting context in which to explore further how discourse contexts and media impact the performance of language activities. Just as conversation analysts turn to exceptional cases to elucidate the normal (Heritage \& Atkinson, 1984), the ways in which Usenet differs from talk can inform our understanding of oral and written language use. By seeing how users adapt to the constraints and opportunities of the medium, one learns more about what is often taken for granted in other channels. Furthermore, the differences which can not be explained by media differences demonstrate other elements of oral and written discourse which may not be apparent. At the same time, Usenet and similar forms of CMC are rapidly growing and extremely popular channels of language use, and the question of how communicators construct their messages in this new medium is intruiging. 


\section{CMC responses -- 11}

\section{Agreement and Disagreement in Usenet}

There has not been any systematic study of disagreement or agreement in CMC. However, the computer has often been accused of encouraging hostile and competitive discourse. The widely noted phenomenon of "flaming"--or attacking others--has been hypothesized to result from "a lack of shared etiquette, by computer culture norms or by the impersonal and text-only form of communication" (Kiesler, Siegel, \& McGuire, 1984, p. 1130). It has thus been argued that the norms governing disagreement in oral interaction are suspended in CMC. Rather than being mitigated, these scholars argue, disagreements are exaggerated. This can be a tempting argument when faced with disagreements such as this one from a newsgroup which discusses the television show Star Trek: The Next Generation (rec.arts.startrek.current, April 12, 1993) ${ }^{1}$. [Note that this post is highly conversational in that incorporates three turns, the first and third by the same speaker]:

\section{Example 1}

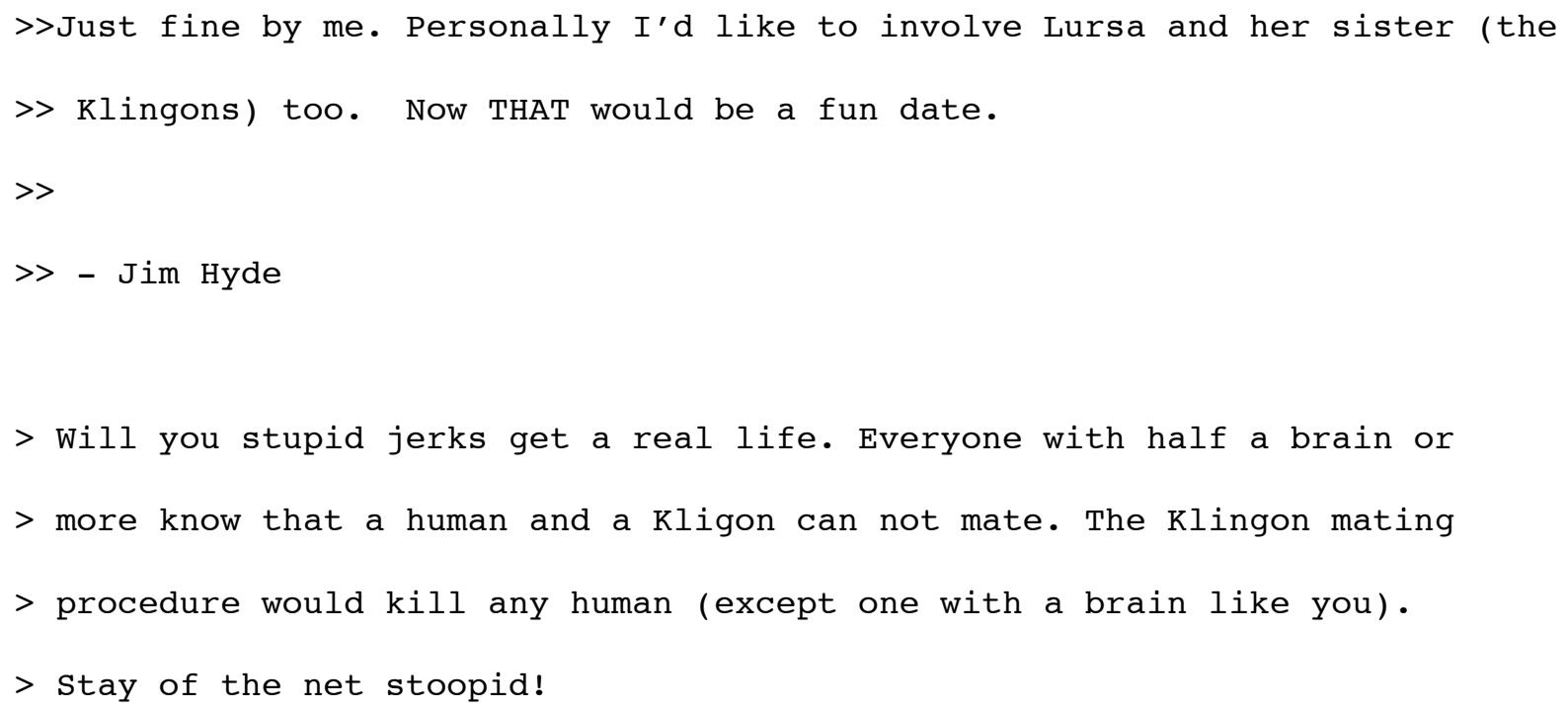




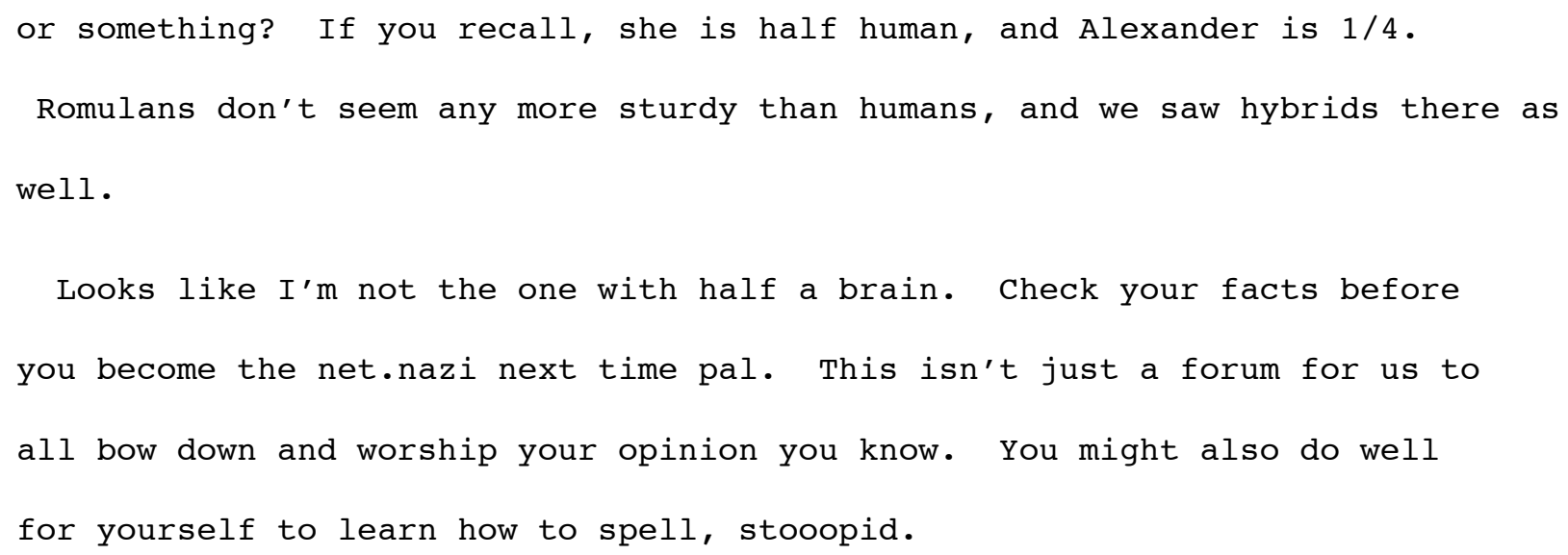

The fact that at least one of the two people in this interaction is male is worth noting as it represents a general feature of Usenet and of gendered disagreement styles. Usenet, like most $\mathrm{CMC}$, is populated by many more men than women, a fact that stems in part from men's greater access to the medium. Since men have greater access, computer-mediated groups, including Usenet, are likely to be exhibit male styles of communication, so that even when women have access, they may not be comfortable or interested in participating. Selfe and Meyer (1991), Ebben (1993), and Sutton (1994) are among those who have shown that many of the gender inequities of face-to-face interaction are perpetuated on-line, where women speak less, are less likely to have their topics pursued, and are seen as dominating when they gain any voice at all.

Disagreement styles may be particularly relevant to creating an online atmosphere in which women are hesitant to participate, as male and female disagreement styles have consistently been shown to be quite different. While men tend toward more aggressive and adversarial tactics, both offline (e.g. Tannen, 1990) and online (Sutton, 1994), women tend toward more community and relationshippreserving strategies in both media (Tannen, 1990, 1994; Herring, 1993; Sutton, 1994). Men, in short, are more likely to be "competition-oriented," while women are more 
likely to be "cooperation-oriented" (Maltz \& Borker, 1992). The participants in the group studied here are $72 \%$ female. This gives us an opportunity to see how disagreement is handled in a computer-mediated group where women dominate and how this affects the group's social environment.

Although flaming is compatable with male disagreement styles, even in maledominated groups flaming is generally considered bad manners. Mabry (in press) analyzed 3,000 messages collected from many forms of CMC and found that more "tense, antagonistic, or hostile argumentative statements" tended to be accompanied by more intense conciliatory behavior. McLaughlin, Osborne and Smith (1995), analyzing a large corpus of messages chastising others' behavior, argue that Usenet standards discourage the wanton insulting or flaming of others. These findings were true of both women and men. Thus, the norms which require strategic articulation of disagreement in oral interaction seem to still be at play in $\mathrm{CMC}$, though they may be instantiated in different ways by men and women.

Agreement and Disagreement in rec.arts.tv.soaps

The agreements and disagreements examined here are part of a broader ethnographic analysis of a newsgroup called rec.arts.tv.soaps (r.a.t.s.). R.a.t.s. was one of the oldest Usenet newsgroups, dating back to 1984. While never one of Usenet's most-read groups, generally ranking between 200th and 300th among Usenet groups in estimated readership (e.g., Reid, 1993), it was one of Usenet's most prolific groups, generating almost 10,000 messages a month. Ultimately r.a.t.s. was so successful that in 1994 it was separated into three groups ${ }^{2}$ to accommodate the ever-increasing traffic. Most of the thousands of participants in r.a.t.s. and its subsequent offspring are female, well-educated and gained their access to Usenet either through their place of 


\section{CMC responses -- 14}

employment or student status. They most often read the group at work or in computer labs.

I collected posts to r.a.t.s. systematically over a ten-month period in 1992. In that time I collected 32,308 messages, $92 \%$ of the total public discourse during those months. I interviewed group members both formally and informally, face-to-face and electronically. I collected responses to two sets of open-ended survey questions posted to the group. I also collected statistical information about events and participants, including how many people participated, how many messages there were, and other macro-level information. The analysis which follows relies almost entirely on the messages themselves, though I draw on survey responses in the conclusion.

I narrowed the data to a coherent but manageable subset by analyzing all the disagreements and agreements in the discussion of one storyline on the soap opera All My Children. That storyline contained 524 messages. An agreement was initially defined as any post which was explicitly responsive to a prior message and which took the same position as that message (though agreements could, and often did, go beyond stating that shared position). Disagreements were defined as those posts which were explicitly responsive to other messages and took positions incompatible with the prior messages. Disagreements were not necessarily directly contradictory, but stated a position which could not logically be held if one held the prior position.

In all but one case, agreements and disagreements were explicitly linked to prior messages through quotation. Such quotations contained an automatically generated reference line indicating the prior writer and were usually edited down to the particular section to which the post responded. This is seen in the bold-faced portion of this agreement from r.a.t.s. (August 13, 1992): 


\section{CMC responses -- 15}

\section{Example 2}

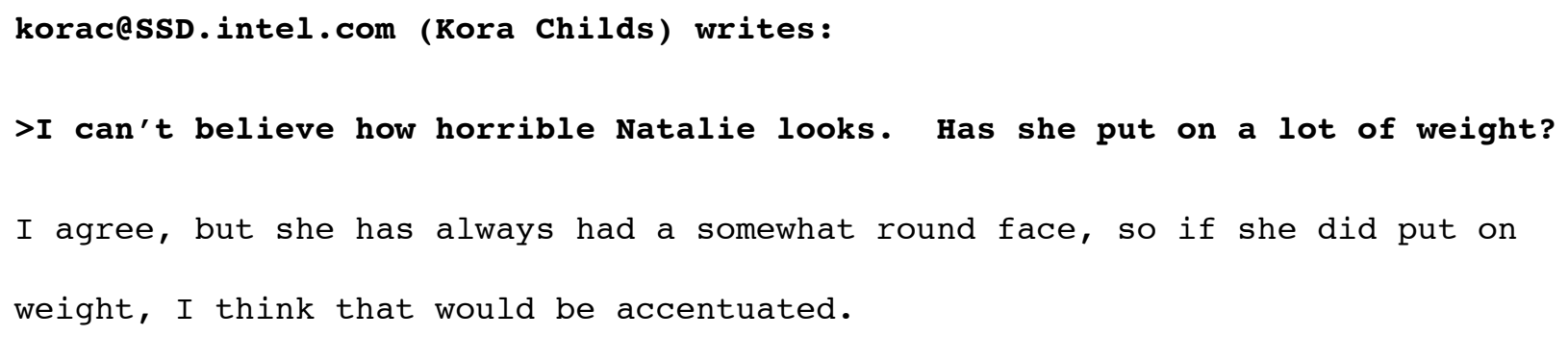

This quotation with reference was used in every disagreement and all but one of the agreements (which was linked through the phrase "as others have pointed out"). There could thus be no question that the authors of these messages were oriented to the prior turns. While these posts may also have done more than agree or disagree, because they directly referenced and either affirmed or contradicted a prior turn they were considered to be agreements or disagreements. This is consistent with the notion that messages are multifunctional and that a single segment can involve multiple activities (e.g. O'Keefe, 1991).

A trained coder and I began by independently coding all of the messages about this storyline as involving either agreement, disagreement or neither. Disagreements between coders were resolved through discussion. The very few cases where we could not agree on a post's status as agreement or disagreement resulted from ambiguous similarity or dissimilarity between the prior and posted positions. Those messages were not counted as agreements or disagreements. Of the 524 messages, most (77\%) were categorized as "neither." These posts covered a wide range of activities, including retellings, new interpretations, questions and answers, trivia, tangents and more, demonstrating that reaching consensus through agreement and disagreement is not this group's central concern. Of the remaining 121 messages, 70 (58\%) were coded as agreements and $51(42 \%)$ as disagreements. 
Since posts, like letters, often cover many topics, the disagreement and agreement responses were often only part of a post. Like Mulkay $(1985,1986)$, the analyses presented here look only at the paragraphs immediately relevant to the topic of disagreement and agreement. I analyzed those sections of posts in which participants agreed or disagreed with a previous position, including all those components of speech which positioned a message as an agreement or disagreement and those which framed these activities. While this move pulls these responses from the full messages, it situates them in the temporal thread of talk in which they were embedded.

I developed a detailed coding scheme for these 121 segments by analyzing them repeatedly to determine what features appeared in multiple messages, a process which involved continual turning from data to categorical scheme, the latter being refined with subsequent re-readings of the former. The resulting scheme had 17 categories, two of which (quotation with reference, and references to others' talk) I have discussed above as methods of demonstrating explicit linkage to previous messages. The remaining 15 were: expression of the "need" to reply, other ways of linking to a prior message, explicit indication of agreement or disagreement, assertions which affirmed or contradicted the prior message, partial agreements, qualifications, elaborations, provision of reasoning, expression of gratitude to the previous poster, apologies, explicit acknowledgment of the other's position, use of the other's name, smiley faces, framing as non-offensive and a catch-all "other" category. I will elaborate on these categories below.

Once this scheme had been generated, the agreements and disagreements were coded for each feature separately by each coder. Messages were coded for the presence or absence of each component rather than for quantity or sequential ordering (although at times sequences were apparent and will be mentioned in the following analyses). There were several differences in our initial codings. To some extent this was due to 
misunderstanding about the definition of the categories. In other cases this resulted from difficulty distinguishing between categories (this was especially true of separating reasoning from elaboration). In some cases the problems stemmed from focusing on different agreement or disagreement responses within the same posts. However, despite our initial divergences, we were able to resolve our coding differences remarkably easily and there were no cases where we still disagreed after discussing the logic of our choices. While this categorical scheme is not the only way one could analyze the message components of these posts, it did account for almost all of the agreement or disagreement-relevant segments. Only one message contained an "other" way of linking to a previous message, and only 7 contained a message component categorized as "other."

Before discussing the features of agreements and disagreements, a brief summary of the storyline is in order. It involved an abusive psychotic man, Carter Jones, who terrorized several women, eventually kidnapping one, Natalie. It was both emotional, especially since many women in the group had been abused, and controversial, since participants enjoyed the actor portraying Carter but resented the story's victimization of women. As a result, the disagreements and agreements seemed especially likely to be loaded with social and personal significance, enhancing any need for strategic articulation and making relevant message features more prominent.

\section{Message Components of Agreements}

According to Pomerantz (1984), conversational agreements tend to be simpler than disagreements, perhaps consisting only of the agreement itself, an upgrade, or a downgrade. In his analysis of letters, Mulkay $(1985,1986)$ found topical situators; agreements were often prefaced by specification of the topic to which they responded. The agreements here looked more like those in letters than like those in oral interaction. Some of the features of each were present, though they contained several features that 
appeared in neither conversations nor letters. Besides those message features which served to create the agreement, there were often prefaces, message features which enhanced social alignment, which mitigated offense and which elaborated on the agreement.

Links to previous discourse. The first part of almost every agreements in this sample was an establishment of a link to an earlier message. As I have discussed above, all but one of the agreements was built off of a quotation with reference. The two other message features which connected agreements to prior turns were used far less frequently. These were referring to previous talk, which was found in eight posts, and expressing the necessity of responding, which occurred in one post where the author said she "had" to respond.

Creating agreement. In many of the posts, agreement was achieved at least in part through explicit use of the phrase "I agree" (as seen in Example 2 above) and strong agreement tokens such as "indeed," "you said it " and so on. These were coded as explicit indicants of agreement. Agreements were marked like this in $67 \%$ of the agreements. Another means of creating agreement was by making an assessment which reiterated the point of the previous post. This category included upgrades and downgrades as well as matching agreements. This example (October 8, 1992), touting the virtues of Natalie's son (twelve-year-old Timmy) and his dog Harold, demonstrates an explicit agreement token and an assessment as well as a reference to previous talk (the position which is being agreed with and the indicants of explicit agreement are in boldface):

\section{Example 3}

>Is it just me, or is Timmy still the smartest person in Pine Valley? Notice >how he immediately caught on that Carter was dangerous? Wasn't he the first 
>to notice his mom was "different" when JaNut was masquerading as Natalie?

Indeed. This has been talked about a lot ... Timmy and Harold are the only ones with a clue in PV! I'll bet if dogs were allowed in the hospital, good old Harold would take a bite out of Carter and drag him down to the station.

These assessment statements appeared in less than half $(41 \%)$ of the agreements.

While the vast majority of agreements were explicitly marked as such, $11(16 \%)$ of the agreements contained neither explicit agreement markers nor this kind of assessment. Instead, they demonstrated similarity in position by elaborating on the other's assessment in a way which assumed the same viewpoint or by providing reasoning for the other's claim. Here is an example (October 14, 1992) in which the poster's elaboration of the prior's claim implies agreement that the adults are treating Timmy with more respect:

\section{$\underline{\text { Example } 4}$}

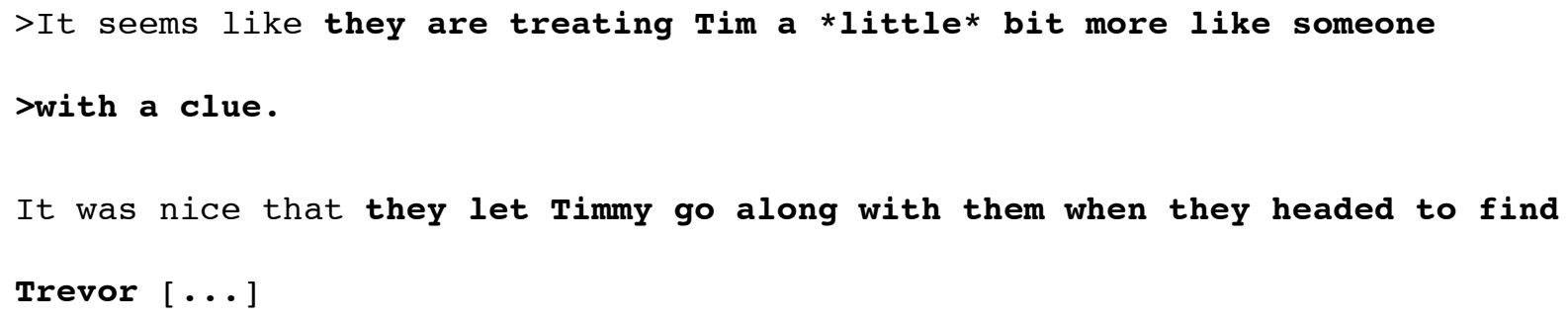

Social aligners. Many agreements contained socially-aligning message features including use of the others' name, acknowledgment of the other's perspective, smiley faces and the expression of gratitude. Participants used the other's name in $21 \%$ of the agreements. Identifying a previous poster by name might seem at first glance to be simply a technique to link one's post to a previous utterance. But most of the time naming was used, the name already appeared in the reference accompanying the quotation. Naming 
CMC responses -- 20

is also a means of addressing a message to a specific recipient. However, were writers intending to address only one person, they could have sent their message via email directly to that individual. One function naming may be serving here is to enhance public recognition of the other. In the often-anonymous terrain of Usenet, where identity cannot be achieved without name recognition, this form of direct address helps build the other's identity. Naming can further be seen as a way of personalizing the group as a whole, populating it with identified individuals.

Respect for the other's position was demonstrated twice by acknowledging the other's perspective with phrases like "I see what you mean." The infrequency is not surprising, given that agreements assume similar, if not identical, perspectives. Another affiliative cue was the smiley face, a socio-emotional cue built out of punctuation marks, usually a colon, a hyphen and a right parenthesis :-). This "emoticon," as such socioemotional cues are called in network slang, is used much as a smile is in face-toface interaction, indicating liking and general good spirits. Smiley faces occurred in eight agreements, many of which were authored by the same woman. The expression of gratitude, where one poster thanks another for expressing their perspective, occurred twice. Though it is rare, the expression of gratitude is an interesting phenomenon because it demonstrates that seeing someone else voice one's own perspective can be satisfying.

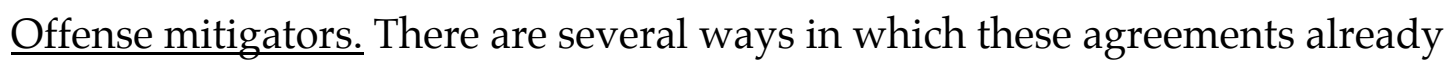
differ from their oral and epistolary counterparts. No agreements in this database consisted of a quoted prior turn followed solely by an explicit agreement marker or an upgrade, downgrade or matching assessment. Several agreements included additional affiliative components, as though the affiliative qualities of agreeing were somehow not enough or did some sort of damage which required compensation. Another way in which these agreements differ from conversational and epistolary agreements is that 


\section{CMC responses -- 21}

almost a quarter $(24 \%)$ of them contained qualifiers, a tactic more commonly associated with differences in position than similarities. Qualifying an utterance modifies the poster's footing by decreasing the extent to which the speaker can be held accountable for its content (Goffman, 1981). The qualifiers used in r.a.t.s. tended to exploit the shift to the subjective with phrases such as "I think that," "that's only my opinion," and so on.

Furthermore, $37 \%$ of the agreements contained reasoning, which (in order to clarify the blurred line between reasoning and elaboration) was operationalized as anything that made more sense as the second half of the sentence "I agree with the quoted utterance because..." than in the sentence "I agree with the quoted utterance and..." In a few cases this reasoning functioned to create the agreement. However, many agreements which were marked explicitly also contained reasoning, another strategy more commonly associated with disagreements.

Elaborations. By far the most striking aspect of agreements was that $84 \%$ of them contained elaborations. Like reasoning, in some cases elaborations were the means of creating agreement in the absence of explicit indicants of agreement (as seen in Example 4), but in most cases occurred along with explicit indicants of agreement. When they occurred with explicit agreements, elaborations moved the talk from the immediate agreement to a new but related angle or topic, as is seen in the inclusion of Harold in Example 3 above. The most common elaborative shift was going from agreement with someone else's evaluation to discussing one's own reaction. Thus people used agreements as a launching pad for putting their own interpretations into the public discourse. Here is an example of this (October 19, 1992): 


\section{Example 5}

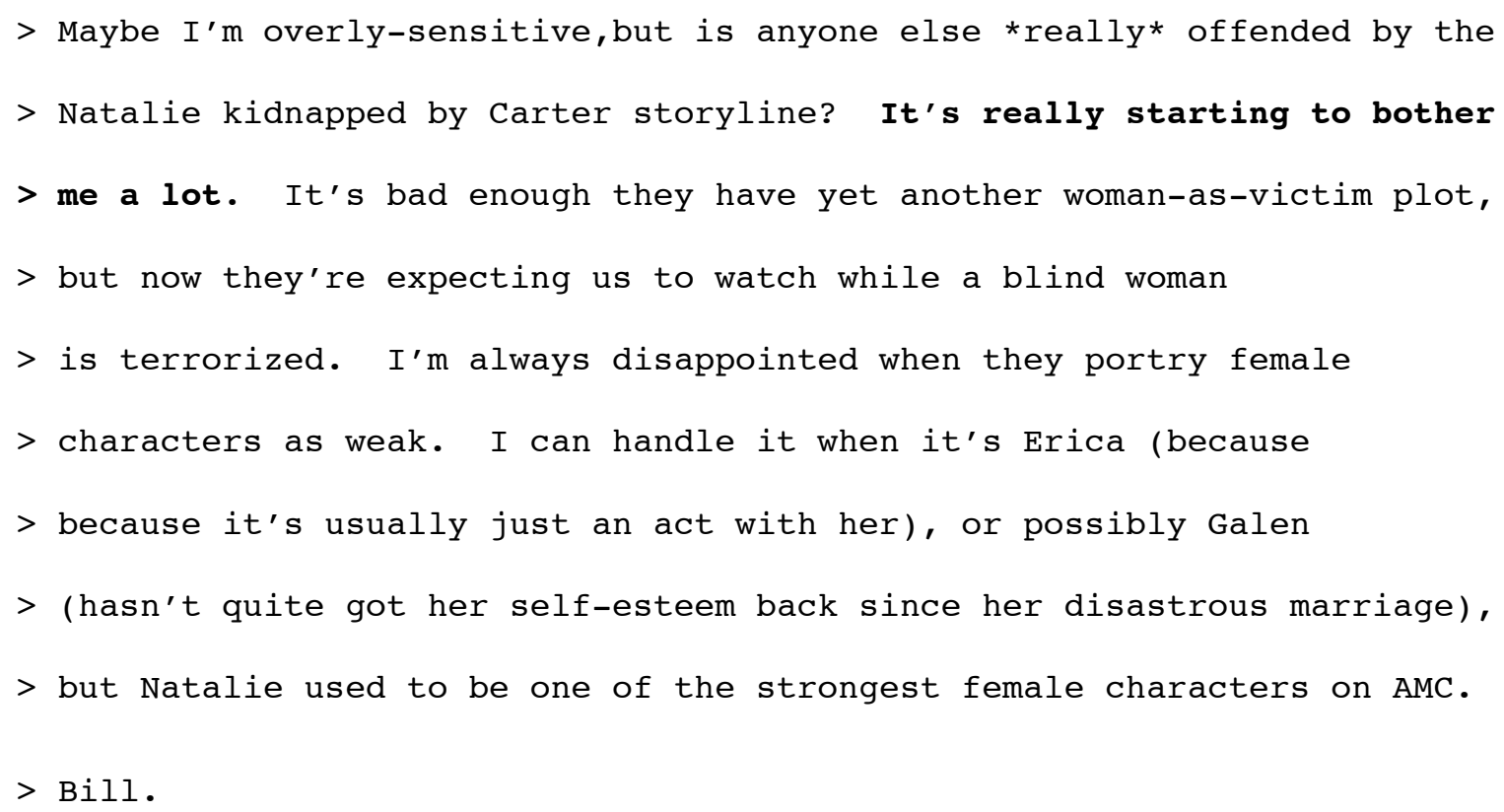

\section{Discussion of Agreements}

These findings provide evidence that simple agreement is insufficient in r.a.t.s. In the full Carter Jones discussion, which had 524 posts, there are only 70 agreements, constituting just $13 \%$. While this is more than the $10 \%$ of the corpus that disagreed, it is still surprisingly little, given that fan communities are built largely on shared meaning (Jenkins, 1992). Only 41\% of agreements included assertions restating the other's point. Furthermore, several of the agreements contain components which actively enhanced the affiliative qualities of agreeing, as though the alignment created by agreement were 
CMC responses -- 23

inadequate to the task at hand. Agreements also incorporated features often associated with disagreement including qualification and reasoning. Finally, even when agreement was made explicit, almost all of the agreements involved some form of elaboration which often brought the respondent's perspective into the discussion. What this suggests is that there is something more to the task at hand than coordinating shared perspective, as is the case in conversational and epistolary agreements. I will return to this after an examination of the group's disagreements.

\section{Message Components of Disagreements}

Like r.a.t.s. agreements, disagreements looked in many ways like their oral and epistolary counterparts. They were more complex and mitigated than were r.a.t.s. agreements. However, like the r.a.t.s. agreements, they contained features which are uncommon in their oral and written counterparts.

Links to previous discourse. Disagreements were linked to prior messages in essentially the same ways that agreements were. All the disagreements used quotation with reference. References to previous talk occurred in $14 \%$ of the disagreements (compared to $11 \%$ of the agreements). Disagreers were somewhat more likely to express the need to reply, but the numerical difference was so slight that it would be inappropriate to draw any firm conclusions from this.

Creating disagreement. As in agreements, disagreement could be created through explicit indicants of disagreement. These were operationalized as the use of the word "disagree" and its synonyms, disagreement tokens such as "but" and through assessments which contradicted the claim of the quoted message. In Example 6 (October $19,1992)$ three women have been debating whether or not Natalie is responsible in any way for her kidnapping. Liz offers an initial viewpoint. Pam disagrees with her. Anne defends Liz's point of view, disagreeing with Pam, and in this post, Pam responds to 


\section{CMC responses -- 24}

both Anne and Liz. After offering an apology, using naming to enhance affiliation (additionally marked with the phrase "my buddy") and framing her response as necessary, Pam explicitly indicates disagreement with both an explicit indicant and a contradictory assessment (these are in bold-face, as is the assessment being disagreed with).

\section{Example 6}

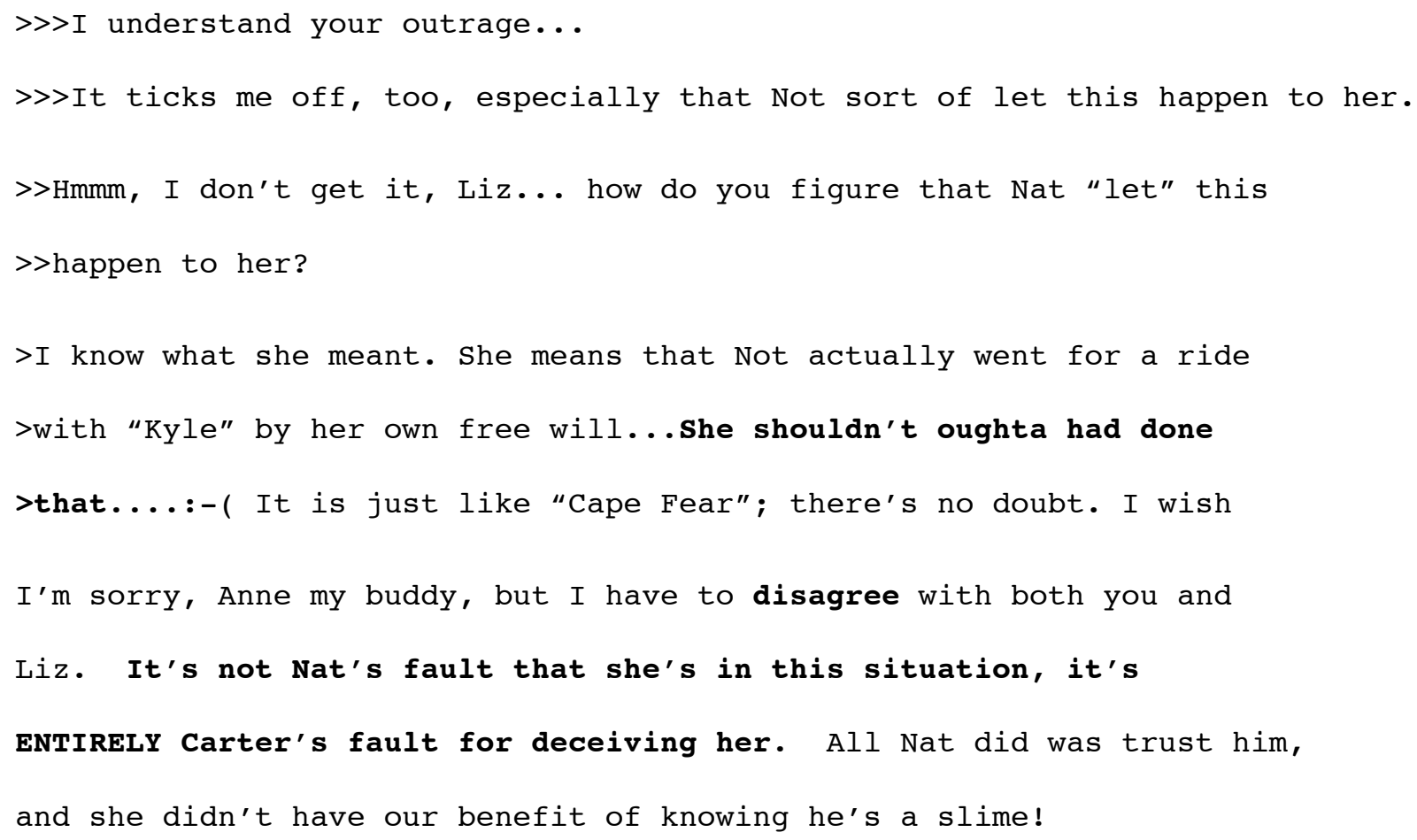

Explicit disagreement markers and contradictory assessments are the most directly confrontational message features evident in this data because they are the least ambiguously oppositional. Explicit markers were used in $47 \%$ of the disagreements, $20 \%$ less often than agreement markers were used in agreements. On the other hand contradictory assessments were far more common in disagreements than were affirming assessments in agreements, occurring in $67 \%$ of the disagreements, versus $41 \%$ of the agreements. 


\section{CMC responses -- 25}

As was the case in agreements, there were some disagreements which did not use these explicit indicants to create disagreement. The $18 \%$ of disagreements which were accomplished implicitly achieved disagreement by providing counter-examples (coded as elaborations), through reasoning which contradicted the claims of the prior message, or by posing questions which clearly challenged the prior claim. This is an example of a disagreement achieved through a challenging question followed by reasoning (August 3, 1992):

\section{Example 7}

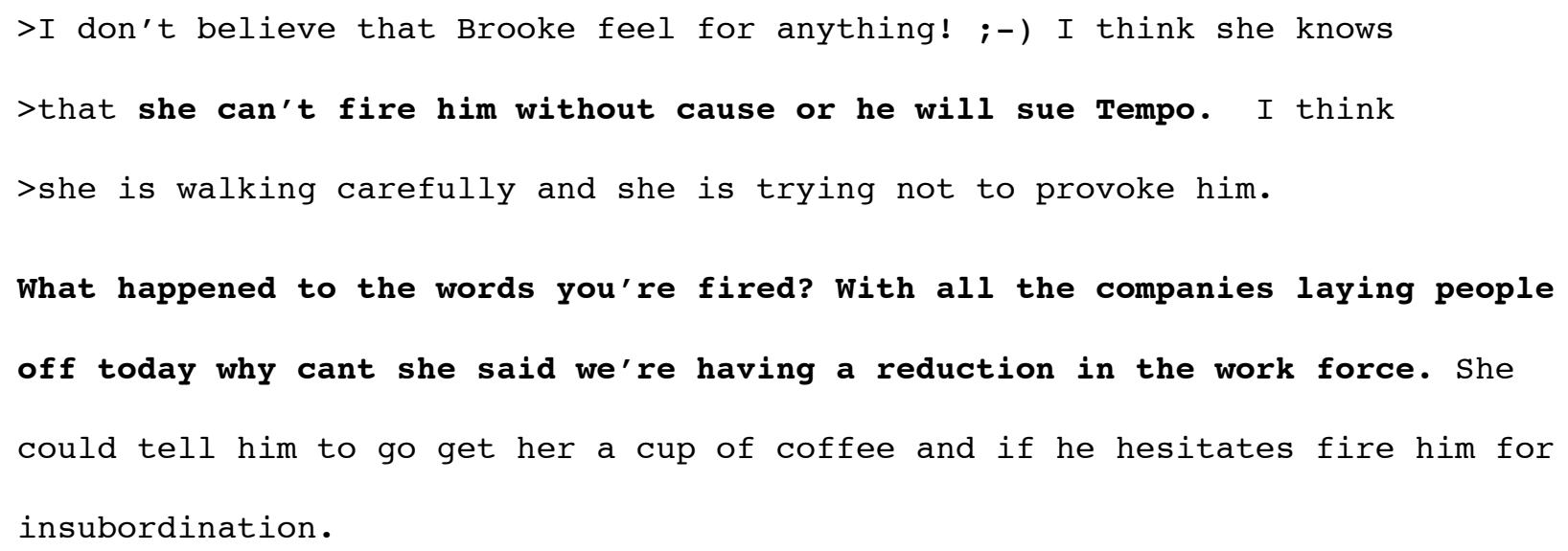

Social aligners. As was the case in both Pomerantz's (1984) and Mulkay's (1985, 1986) analyses, many disagreements in r.a.t.s. (29\%) were prefaced by partial agreements. Partial agreements were generally followed by disagreement tokens such as "but" and "though" or phrases such as "at the same time" positioning what followed as disagreement. In Examples 8 (October 2, 1992) and 9 (October 20, 1992) this disagreement strategy can be seen. In Example 8, the dispute is over whether or not Brooke was tough enough when she fired Carter after catching him using Tempo magazine resources to develop potentially-incriminating photographs of Galen hugging Trevor. The quoted claim is that Brooke handled herself well. The response never states "Brooke did not handle herself well," (i.e., it does not have a contradictory assessment) 


\section{CMC responses -- 26}

but instead agrees initially (which is boldfaced), uses an explicit disagreement marker ("but”), then describes behavior of Brooke's which suggests she did not deal with the situation adequately:

\section{Example 8}

>Well, it seems she got some QUALITY time in yesterday, firing Carter Jones! >Go Brooke! Brainslap of the Week! She held up to him and didn't back off >or squirm! I loved it...:-)

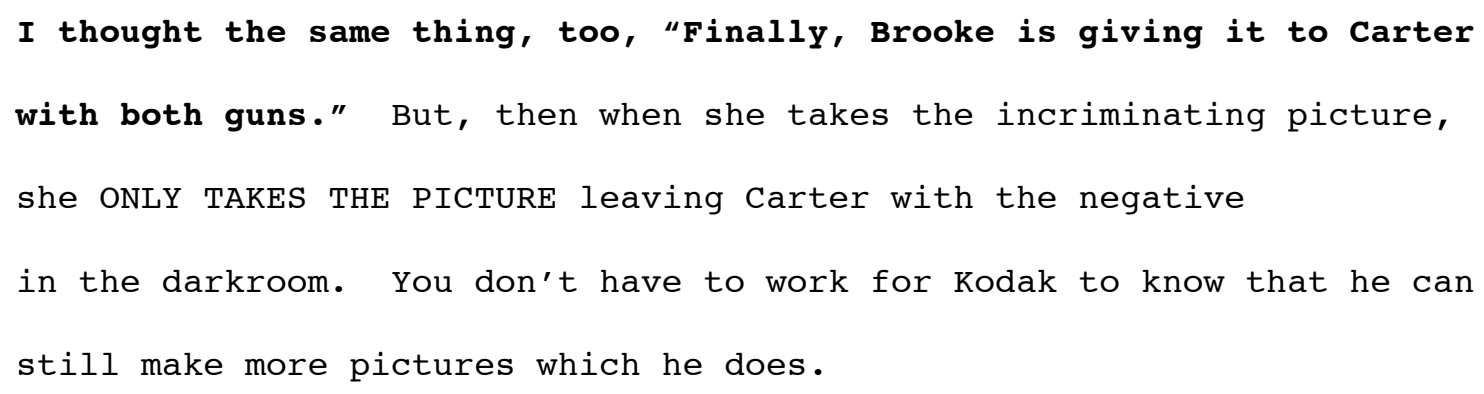

We see the same pattern here in a disagreement over whether or not kidnapped and blind Natalie was handling Carter as well as she could:

\section{Example 9}

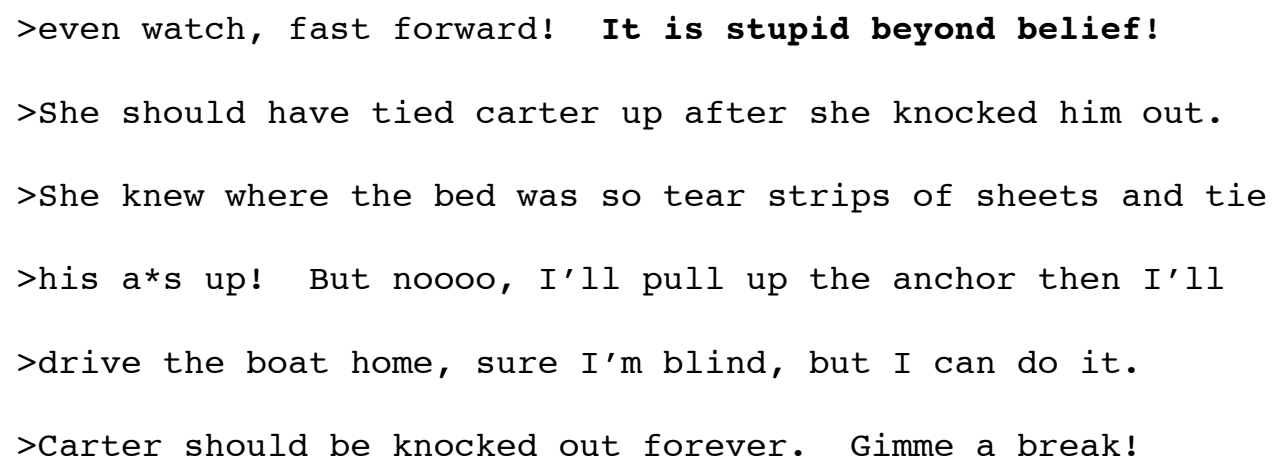


-- to try and make Carter dislike her, since he only likes weak and dependent women. She seemed to try and play his psychological mind-f**k games right along with him. I was impressed, too bad it didn't work.

Another notable aspect of both these messages, and of these disagreements in general, is that the partial agreements often indicated a temporal shift in the posters' thinking. Initially they held the same position ("I thought the same thing too, but then..." and "At first ... But watching her in action on Monday's show..."), but then they came to interpret soap events differently. The disagreement is therefore situated as one with their earlier selves as well as with the other, enhancing the alignment between self and other.

Naming was also used to create alignment in disagreements, as can be seen in this excerpt from Example 6, in which the poster makes explicit the affiliative quality of naming with the phrase "my buddy":

I'm sorry, Anne my buddy, but I have to disagree with both you and Lisa....

Interestingly, naming was less likely in disagreements than agreements, occurring only $18 \%$ of the time. While specific identification of another would serve to create recognition in disagreements as well as in agreements, it could be that doing so with disagreements might create negative recognition, thus doing more of a disservice than service.

Participants acknowledged the perspective of the other in $6(12 \%)$ of the disagreements, a move they made only twice in agreements. Here (October 21, 1992) Liz responds to Pam's contradiction by acknowledging Pam's position before disagreeing (Pam's message is quoted with a colon rather than the usual " $>$ " in the left margin, presumably a feature of Liz's newsreader). Note also the explicit disagreement marker ("however") and the use of naming: 


\section{CMC responses -- 28}

\section{Example 10}

[stuff where Anne and Liz say Nat kinda put herself in this bad situation deleted ]

: ENTIRELY Carter's fault for deceiving her. All Nat did was trust him, : and she didn't have our benefit of knowing he's a slime!

:

: I know that neither of you meant anything by what you said, but I

: couldn't let it go -- it was too close to Carter's rationalizing that

: the women he beat were responsible for the beatings, not him.

I see what you are saying, Pam.

However, some poster wrote something about Nat not forgiving Trevor for not rescuing her sooner - and if you look at it that way, I have to say that Nat would have no reason to hold this against Trevor since the whole mess was caused primarily by Carter's mental illness and secondarily by Nat's opening up to him / agreeing to leave the hospital with him. The whole thing could never have happened if Nat hadn't left the hospital instead of waiting for Timmy. Of course, I suppose Kyle could have abducted Natalie more subtley - like wheeling her out for some xrays and just continuing right on out of the hospital!

I am not saying that Nat deserved what happened to her or that she was responsible for the beatings - just that if had she been less trusting she wouldn't have made Carter's abduction plan so easy, but then again it was her blindness (Carter's fault entirely) which which made it all possible.

I know it is annoying when people must be less trusting or re-arrange their own lives or personalities just to be able to live in a society 
full of criminals and psychos, but it is a part of life. I know this pretty well from living in the big city after growing up in a small town. And being a 5'2" woman doesn't help at all!

Smiley faces were not used often in disagreements, occurring in only 8 cases, just as they went unused in most agreements. For all the attention they have received in the popular media, smiley faces are simply not an important cue in these activities.

Offense mitigators. Almost two thirds of the disagreements (61\%) included the provision of reasoning to support the writer's perspective, a common feature of conversational disagreements. In this example (August 3, 1992), the contradictor supports her position with evidence. The additional reasons are boldfaced:

\section{Example 11}

>After watching Thursday's episode with Stephen and Carter I have to ask a >couple of things: 1) Why is it that ALL soaps think women can't take $>$ care of their own problems and need some man to totally blow things for >them?

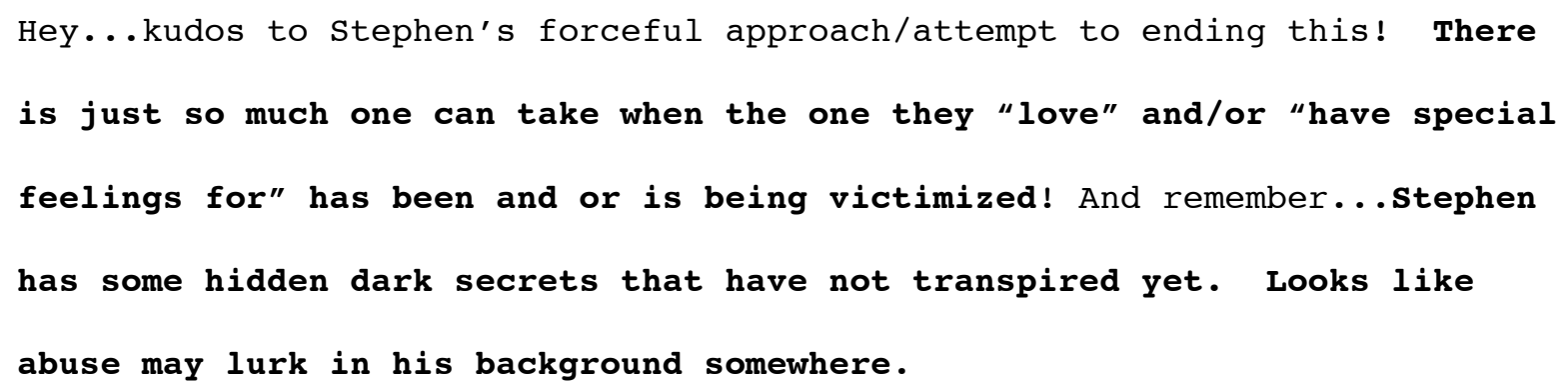

Qualifications were also common in disagreements, occurring in $43 \%$ of them. Qualifiers frame disagreements as resulting from differences in subjective opinion, thus leaving room for the other's viewpoint. Here is an example (July 23, 1992) of a dispute 


\section{CMC responses -- 30}

that uses such qualifying markers prior to and following the contradictory assessment (the qualifications are in bold face):

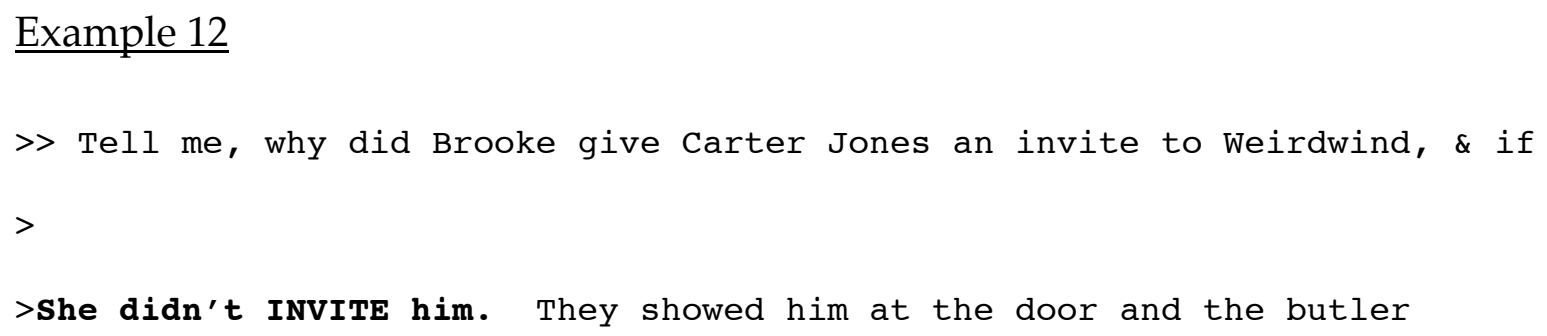

While apologies were not particularly common in disagreements, they were moderately $(7 \%)$ more frequent there than in agreements. Example 6 above demonstrates the apology:

I'm sorry, Anne my buddy, but I have to disagree with both you and Liz...

The final mitigating strategy that occurred in disagreements was framing as nonoffensive. This technique, used four times, is when the poster explicitly keyed her activity as something other than confrontational. In one case this involved prefacing a contradictory assessment with "I think this is so funny." In another case someone wrote "no offense to Knot's Landing" just before suggesting that Cape Fear had been a greater influence on the story line. 


\section{CMC responses -- 31}

Elaborations. As was the case in agreements, next to quotation, the most common message feature of disagreements was elaboration, which occurred in $69 \%$. The last paragraph of Example 10 above is an instance of elaboration in disagreement. While their function in agreements (where they were more common) is not immediately apparent, their use in disagreements could be seen as a way to mitigate, as they move the discussion away from the immediate point of conflict. However, the fact that they are so common in this data, yet have not been noted in oral or epistolary disagreement, suggests that they are probably serving further functions as well.

\section{Discussion of Disagreements}

There are a number of similarities between these disagreements and agreements in r.a.t.s. They are not particularly common, constituting only $10 \%$ of the total messages about Carter's storyline. They are overwhelmingly linked to previous messages using quotation with reference. Next to this linking mechanism, the most common message feature of both was elaboration. In general, both disagreements and agreements were built out of the same set of message features, though in differing proportions.

In contrast to agreements, disagreements contained more mitigation. They were more likely to have reasoning, to be qualified, to apologize, to acknowledge the other's perspective, and to be framed as non-offensive. Interestingly, they contained more contradictory assessments than agreements contained affirming assessments. While only $41 \%$ of agreements had assessments which reiterated the point of agreement, $67 \%$ of the disagreements contained assessment which directly opposed the position embedded in the quoted material. One interpretation of this is that it was easier to express disagreement strongly because the written medium distanced writers from those they challenged or otherwise lessened their confrontational impact. Were this the case, however, one would not expect to see such enhanced use of mitigating strategies in comparison to agreements. Another possibility is that the medium made it less clear 
CMC responses -- 32

when something should be interpreted as disagreement, thus the need to mark something as disagreements would be enhanced. This explanation is also suspect given that disagreements were less likely to be marked than agreements through use of the term "disagree" and other explicit tags. Again, these qualities of r.a.t.s. disagreements, in conjunction with the fact that so many disagreements contained elaborations (though less so than agreements), suggests that disagreement, like agreement, is being used for functions other than the interpersonal ones seen in oral and epistolary disagreement.

\section{Discussion}

These findings are in many ways consistent with work on agreement and disagreement in oral conversation and in writing. In general, agreement appears to be easier to perform while disagreement requires more strategic mitigation. However, there are several notable differences between the way these activities are done orally or in letters. In both agreements and disagreements quotation is ubiquitous; there are fewer secondary assessments than one might expect; most strikingly, there is pervasive elaboration. Agreements further differed from their oral and epistolary counterparts in their frequent use of reasoning and qualification.

Many of the differences between these Usenet messages and their oral counterparts are due to the hybrid nature of this particular medium. Quotation, as I have suggested above, takes advantage of the fact that messages are electronically stored in order to mark topic and create an orientation to particular prior turns. This mitigates the medium's spatio-temporal separation while keeping it fairly conversational. The use of quotation may partially explain why there aren't more secondary assessments, particularly in agreements. Quoting a previous statement wordfor-word can serve as a secondary assessment in the same way that a restatement of an assertion would in face-to-face conversation. 
CMC responses -- 33

Next to quotation with reference, the most common feature of both agreements and disagreements was elaboration. Previous work does not indicate that elaboration is particularly common in the oral versions of these activities. While letters do involve multiple topics, that is a distinct phenomenon from these elaborations, which are grafted directly on to the agreement or disagreement. Elaboration can be seen as a way to increase a message's interest value for a mass audience, meeting a wide readership's needs while demonstrating one's own competence at doing so. Agreements without elaboration bring nothing new to the discussion. Indeed, the prevalence of elaboration indicates that agreement is used more for the purpose of making one's own novel contributions relevant to the ongoing discussion than a means of coordinating perspective.

The importance of novelty in contrast to coordination is seen in the survey responses. When asked in surveys what the group's norms are, the answers often included comments like "the [posts] that follow-up a post with 'i agree' and nothing else are boring" and "don't answer a posting with 200 lines by including the entire original article and only adding a 'me too'"(Kelly, November 27, 1991; Jennifer, December 13 1991). Similarly, one of the most common reasons people gave when I ask why they don't post is that they had nothing "to add" but "me too" and didn't want to "waste bandwidth." The use of the "bandwidth" metaphor implies the mass media concerns to which speakers must orient in Usenet. This taboo on repetition was also found in a survey of r.a.t.s. and other Usenet groups by McLaughlin et al. (1995).

Just over two thirds of disagreements also involve elaboration, suggesting that even when writers are countering previous points they are still obligated to move the discussion in novel directions. The presence of reasoning in so many agreements can also be understood as a means of introducing new material into the ongoing discussion. Similarly, qualifying an agreement adds the angle of a new subjective interpretation. 
The emphasis on novel contributions seen in elaboration and limited use of affirming assessments is further explained by features of the institutionally-situated context of Usenet use. Participants are often reading during breaks in work and have limited time to devote to the group. If one is going to impose upon the readers' time by contributing a post, that post needs to offer something previous posts have not.

The need to avoid redundancy and increase diversity gains added significance when understood in the context of the institution around which this group has organized, the serial soap opera. Allen (1985) argues that soap operas are over-coded, conveying far more possible meanings than the story's progress requires. This openness to multiple meanings is one of the primary qualities which attracts the genre's viewers. The game of soap viewing is in bringing expertise and knowledge to bear on decoding these meanings; the more meanings one can create, the more interesting the show. Collaboration in this game, as happens in r.a.t.s. discussions, provides resources that allow all participants to build richer interpretations than they can generate alone. Since this enhances the fundamental pleasure of being a soap opera fan, language practices are oriented toward maximizing the contribution of new interpretive resources. The diversity of viewpoints presented in r.a.t.s. is frequently cited as one of its primary appeals, as seen in survey responses like "it's fascinating to see all the different points of view on such a range of topics" (Jamie, October 1,1993) or in the descriptions of "successful" posts as those which "are either people's own views on what happened on a soap opera, or new plot ideas" (Kelly, December 2, 1991). Disagreement invariably involves the contribution of different interpretive resources, and thus facilitates the group's primary goal. This helps to explain why there are more contradictory assessments in disagreements than affirming assessments in agreements.

An understanding of the centrality of soap opera's contribution to the discourse's structure as well as its content also furthers our understanding of why, even though 
CMC responses -- 35

they broaden the interpretive resources, disagreements are handled with such tact. In comparison to the disagreement from rec.arts.startrek.current quoted above, disagreements in this group are remarkably civil. They always remained focused on differences in positions, rather than shifting to personal attacks. In Goffman's (1967) terms, there was no "aggressive facework;" in netiquette terms, there were no flames. The call for tact is related to the nature of topics which arise in making sense of soap operas. Because soap operas revolve around emotions and relationships, their interpretations often rest on issues which are emotionally-loaded for the participants, and which are not usually discussed in public. These discussions call for both emotional identification and, often, private self-disclosures. If people who posted interpretations imbued with their private experience were attacked, they and others would quickly stop posting their ideas. The group's efforts at sustaining a respectful environment can be seen as a response to this challenge; by creating an emotionally welcoming space, they decrease the threats to people with something relevant but private to self-disclose. The most dramatic instance of this sort of interpretation in this sample were those responses to Carter's beatings in which posters' interpretations rested on their own experiences as abused spouses. Posts like this were invaluable for those who had never had to face abuse in their own lives both in understanding and evaluating the characters' behavior. That people be able to post such things is therefore very much in the interest of the community, generating strong motivation to frame disagreements with qualifications, acknowledgments of others' perspectives, reasoning, and other mitigating and affiliative devices.

The high level of civility on r.a.t.s. can also be attributed to the overwhelmingly female population. The disagreement style seen in this group is consistent with previous work on women's language showing a strong orientation toward community preservation at the same time one's own point is advanced. Several survey respondents 


\section{CMC responses -- 36}

made this argument. One woman (Jane, November 27, 1991) attributes the group's tact is attributable to a combination of the group's topic and gender balance, writing: "This group is generally much more civil than most others. Perhaps soaps don't lend themselves to flame wars [...] Perhaps it's because of the unusually high (for USENET) ratio of female to male posters." The civility of r.a.t.s. disagreements results in an online space where, as part of of discussing soap operas, women are able to discuss real-life issues of fundamental importance to them without the threats of verbal attack rampant elsewhere on Usenet (see Ebben, 1993, for a discussion of how men antagonized women discussing similar issues on the newsgroup soc.women).

\section{Conclusion}

I have argued that the message features in r.a.t.s. agreements and disagreements stem from five interrelated factors -- the Usenet medium, the institutional context of work, the topic under discussion, the participants' gender, and the social context participants strive to create. In closing I want to suggest how these findings can influence our understandings of Usenet and of oral and written interaction.

As this study makes clear, one cannot overlook the importance of media in influencing the performance of agreements, disagreements, or any other language activity. The features seen in this analysis strongly reflect the particular demands of Usenet, demands which stem from its status as a hybrid of spoken and written, interpersonal and mass communication. Usenet, and other asynchronous public online forums, requires that its participants actively work to invoke the previous contexts on which their contributions are built. Usenet writers must also orient toward the concerns of a large distributed group as well as those of a single interaction partner. While the medium through which this is done is writing, the interactive and impermanent qualities of Usenet render the interaction akin to orality. There are a number of varieties 


\section{CMC responses -- 37}

of CMC, including private email, semi-private mailing lists, synchronous dyadic and multi-party groups, and others. A full understanding of the ways in which the computer medium affects language use will depend on systematic comparison within and across these different modes of CMC.

It would be a mistake to assume that it is only in unusual or hybrid media such as this that the medium impacts language use. Just as topical prefaces and delays in this data are responses to the asynchronous written medium, the lack of topical prefaces and uses of time in oral conversation are responsive to the oral medium. The taken-forgranted qualities of speech and print should not blind us to the ways in which our verbal behaviors are shaped by media possibilities and constraints.

This study also affirms the importance of bringing context to bear on making sense of how language activities are structured. The challenges and opportunities lodged in the activities of agreeing and disagreeing are only fully comprehensible when the participants (in this case their gender), the institutions (in this case Usenet and soap operas), and the genre (here soap opera discussion) are taken into account. Each of these contexts influences linguistic structures in r.a.t.s. Systematic comparisons between language activities across contexts cannot be developed unless the contexts of oral interactions and instances of writing are considered.

Finally, I have claimed that the ways in which agreements and disagreements are structured in r.a.t.s. reflects not only the contexts in which the discourse is embedded, but also the context participants strive to create. Were the group not so invested in having an environment which encourages the expression of a diversity of viewpoints on private topics, many of the structural features we see in their agreements and disagreements might well not exist. It is their orientation toward creating this diverse and friendly environment, as well as toward maintaining it, that shapes these activities. 


\section{CMC responses -- 38}

Though this becomes more obvious in this interactive written medium, it is very much a feature of oral interaction and should not be overlooked there. It is also important to consider the extent to which the structure of activities performed in other written media are responsive to constraints on what type of emergent context is desired.

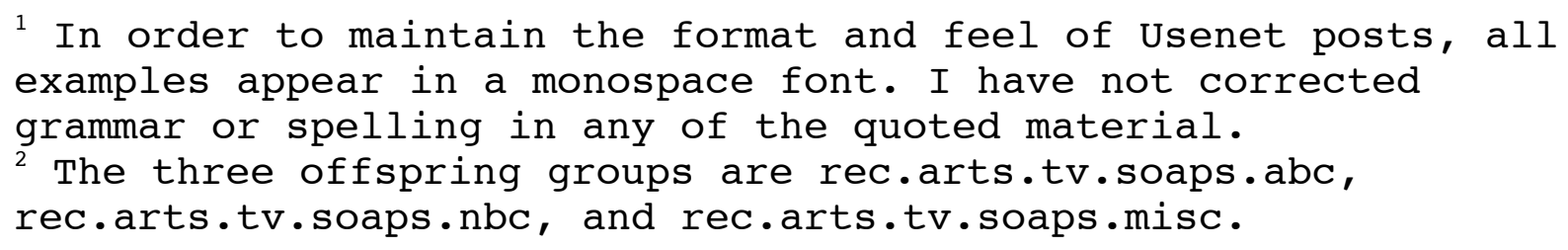




\section{References}

Allen, R. C. (1985). Speaking of soap operas, Chapel Hill: University of North Carolina.

Atkinson, J. M., \& Heritage, J. (1984). Preference organization. In J. M. Atkinson, \& J. Heritage (Eds.), Structures of social action: Studies in conversation analysis, (pp. 5356). Cambridge: Cambridge University.

Basso, K. (1974). The ethnography of writing. In R. Bauman, \& J. Sherzer (Eds.), Explorations in the ethnography of speaking, (pp. 425-432). Cambridge: Cambridge University Press.

Baym, N. (1995). The emergence of community in computer-mediated communication. In S. Jones (Ed.), Cybersociety: Computer-mediated communication and community, (pp. 138-163). Thousand Oaks, CA: Sage.

Biber, D. (1986). Spoken and written textual dimensions in English: Resolving the contradictory findings. Language, $62,384-414$.

Brown, P., \& Levinson, S. (1987). Politeness: Some universals in language usage. Second Edition. Cambridge: Cambridge University.

Chafe, W. (1982). Integration and involvement in speaking, writing and oral literature. In D. Tannen (Ed.), Spoken and written language: Exploring orality and literacy, (pp. 35-54). Norwood, NJ: Ablex.

Danet, B. (1993). Books, letters, documents: The changing materiality of texts in late print culture [On-line]. Available: msdanet@pluto.mscc.huji.ac.il. 


$$
\text { CMC responses -- } 40
$$

Ebben, M. (1993). Women on the net: An exploratory study of gender dynamics on the soc.women computer network. Paper presented at the annual meeting of The Organization for the Study of Communication, Language and Gender, Tempe, AZ.

Ferrara, K., Brunner, H., \& Whittemore, G. (1991). Interactive written discourse as an emergent register. Written Communication, 8, 8-34.

Goffman, E. (1967). On Face-work. In E. Goffman, Interaction ritual, (pp. 5-46). New York: Pantheon.

Goffman, E. (1981). Footing. In E. Goffman Forms of talk, (pp. 124-159). Philadelphia: University of Pennsylvania.

Heritage, J., \& Atkinson, J. M. (1984). Introduction. In J. M. Atkinson, \& J. Heritage (Eds.), Structures of social action: Studies in conversation analysis, (pp. 1-16). Cambridge: Cambridge University.

Herring, S. (1993). Gender and democracy in computer-mediated communication. Electronic Journal of Communication, 3(2).

Jenkins, H. (1992). Textual poachers: Television fans and participatory cultures. London: Routledge.

Kiesler, S., Siegel, J., \& McGuire, T. W. (1984). Social psychological aspect of computer-mediated communication. American Psychologist, 39, 1123-1134.

Kotthoff, H. (1993). Disagreement and concession in disputes: On the context sensitivity of preference structures. Language in Society, 22, 193-216.

Levinson, S. C. (1983). Pragmatics. Cambridge: Cambridge University. 
Mabry, E. (in press). Frames and flames: The structure of argumentative messages of the 'net. In S. Rafaeli, M. McLaughlin, \& F. Sudweeks (Eds.), Network and netplay: Virtual groups on the internet, Cambridge, MA: AAAI/MIT.

Maltz, D. N., \& Borker, R. A. (1982). A cultural approach to male-female miscommunication. In J. Gumperz (Ed.), Language and social identity, (pp. 195-216). Cambridge: Cambridge University.

McLaughlin, M. L., Osborne, K. K., \& Smith, C. B. (1995). Standards of conduct on Usenet. S. Jones (Ed.), Cybersociety: Computer-mediated community and communication, (pp. 90-111). Thousand Oaks, CA: Sage.

Mulkay, M. (1985). Agreement and disagreement in conversations and letters. $\underline{\text { Text }} \underline{5}, 201-227$.

Mulkay, M. (1986). Conversations and texts. Human Studies, 9, 303-321.

O'Keefe, B. J. (1991). Message design logic and the management of multiple goals. In K. Tracy (Ed.), Understanding face-to-face interaction, (pp. 131-150). Hillsdale, NJ: Lawrence Erlbaum Associates.

Ong, W. (1982). Orality and literacy: The technologizing of the word. London: Methuen.

Pomerantz, A. (1984). Agreeing and disagreeing with assessments: Some features of preferred/ dispreferred turn shapes. In J. M. Atkinson, \& J. Heritage (Eds.), Structures of social action: Studies in conversation analysis, (pp. 57-101). Cambridge: Cambridge University.

Reid, B. (1993, August 6). Usenet readership report for July, 1993. news. lists, Usenet. 
Selfe, C., \& Meyer, P. (1991). Testing claims for on-line conferences. Written Communication, 8, 163-192.

Sheldon, A. (1992). Conflict talk: Sociolinguistic challenges to self-assertion and how young girls meet them. Merrill-Palmer Quarterly, 38, 95-117.

Sutton, L. (1994). Using USENET: Gender, power, and silence in electronic discourse. Proceedings of the Twentieth Annual Meeting of the Berkeley Linguistics Society. Berkeley: Berkeley Linguistics Society.

Tannen, D. (1990). You just don't understand. New York: Ballantine.

Tannen, D. (1994). Gender and discourse. New York: Oxford University.

Wilkins, H. (1991). Computer talk: Long-distance conversations by computer. Written Communication, 8, 56-78. 


\section{Author's Note}

An earlier version of this study appeared in my doctoral dissertation at the University of Illinois, Urbana-Champaign, which was directed by Dr. Peggy Miller. I wish to acknowledge her help in directing the research and shaping my understanding of the findings. I also wish to thank Mark Huglen for his work coding the data. 